\title{
Rigid Urea and Self-Healing Thiourea Ethanolamine Monolayers
}

\author{
Cristina Stefaniu, ${ }^{\dagger}$ Pierre-Léonard Zaffalon, ${ }^{\ddagger, \S}$ Alessio Carmine, ${ }^{\|}$Quentin Verolet, ${ }^{\ddagger, \S}$ Samuel Fernandez, ${ }^{\ddagger, \S}$ \\ Tomasz A. Wesolowski, ${ }^{*}, \|$ Gerald Brezesinski, ${ }^{* \dagger}$ and Andreas Zumbuehl*, ${ }^{*}, \S$ \\ ${ }^{\dagger}$ Max Planck Institute of Colloids and Interfaces, 14476 Potsdam, Germany \\ ${ }^{\ddagger}$ Department of Chemistry, University of Fribourg, Chemin du Musée 9, 1700 Fribourg, Switzerland \\ ${ }^{\S}$ National Centre of Competence in Research in Chemical Biology, University of Geneva, Geneva, Switzerland \\ "Department of Physical Chemistry, University of Geneva, Quai Ernest-Ansermet 30, 1211 Geneva 4, Switzerland
}

\begin{abstract}
A series of long-tail alkyl ethanolamine analogs containing amide-, urea-, and thiourea moieties was synthesized and the behavior of the corresponding monolayers was assessed on the Langmuir-Pockels trough combined with grazing incidence X-ray diffraction experiments and complemented by computer simulations. All compounds form stable monolayers at the soft air/water interface. The phase behavior is dominated by strong intermolecular headgroup hydrogen bond networks. While the amide analog forms well-defined monolayer structures, the stronger hydrogen bonds in the urea analogs lead to the formation of small three-dimensional crystallites already during spreading due to concentration fluctuations. The hydrogen bonds in the thiourea case form a two-dimensional network, which ruptures temporarily during compression and is recovered in a self-healing process, while in the urea clusters the hydrogen bonds form a more planar framework with gliding planes keeping the structure intact during compression. Because the thiourea analogs are able to self-heal after rupture, such compounds could have interesting properties as tight, ordered, and self-healing monolayers.

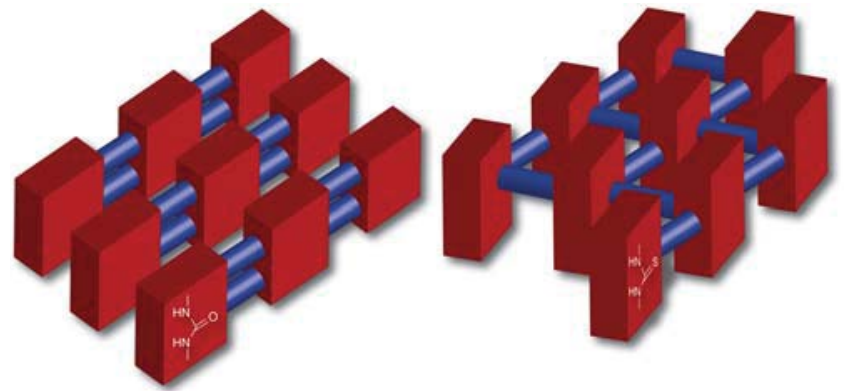

\section{INTRODUCTION}

$\mathrm{N}$-acylethanolamines (NAEs) are naturally occurring singlechain amphiphiles with a surprisingly rich spectrum of medical applications ranging from analgesic, antibacterial, anti-inflammatory, and antiviral to neuroprotective properties. ${ }^{1-3}$ The biophysical properties of the NAEs are equally interesting showing a high propensity to form bilayer-type structures with strong hydroxy group hydrogen-bonding networks and equally strong intermolecular amide $\mathrm{N}-\mathrm{H}$ and $\mathrm{C}=\mathrm{O}$ hydrogen bonds. $^{4-7}$ NAEs can serve as readily accessible model compounds for studying intermolecular forces found in more complex phospholipid systems. ${ }^{8}$ The present study aims to explore the effects of exchanging the amido units with urea and thiourea moieties.

Ureas are useful as structural moieties in supramolecular chemistry or organogelators as they reliably form onedimensional, bifurcated hydrogen-bonding networks linking the acceptor (carbonyl-oxygen) to the two donors (nitrogens) of the neighboring molecule., ${ }^{9,10}$ Thioureas on the other hand show a more complex behavior due to the reduced electronegativity of a sulfur atom compared to the smaller carbonyloxygen, the higher acidity of the $\mathrm{NH}$ protons in ureas compared to thioureas, ${ }^{11}$ and the longer hydrogen bonds caused by the larger size of a sulfur atom and its more diffuse electron cloud. ${ }^{12}$ Moreover, thioureas form different rotamers and the molecules arrange almost orthogonally to each other. ${ }^{13}$
Langmuir monolayers are monomolecular films of insoluble amphiphiles (molecules consisting of a hydrophobic (tail) and a hydrophilic part (head)) on the surface of a liquid. Such twodimensional (2D) systems as well as three-dimensional (3D) lipid vesicles are often used as models to simplify complex biomembranes for a better understanding of structure-function relationships on a molecular level. ${ }^{14,15}$ The monolayer stability at the air/water interface has been already characterized for some urea and amide derivatives. ${ }^{16}$ An important observation was the direct transition (resublimation) of the urea derivatives from the gas-analogous phase to a condensed phase (LC) without going through a liquid-expanded (LE) phase. Amides, however, show both LE and LC phases as well as their coexistence in a plateau region. As for other amphiphiles, temperature and chain length are the important factors: for a $\mathrm{C} 15$ chain, the resublimation was observed at $20{ }^{\circ} \mathrm{C}$, whereas the reduction of the chain length by one methylene unit or a temperature increase lead to the appearance of the LE phase and the first-order LE/LC phase transition. ${ }^{16}$

Here, we present a series of amido, urea, and thiourea ethanolamines in order to assess the importance of hydrogen bonds formed between the hydrophilic head groups on the 
phase behavior of lipid monolayers at the soft air/water interface.

\section{EXPERIMENTAL SECTION}

Materials and Analytical Methods. The starting compounds and solvents were purchased from Sigma-Aldrich/Fluka or Acros and were used without further purification. Column chromatography was carried out using 230-400 mesh silica gel. TLC plates were developed with $\mathrm{KMnO}_{4} \cdot{ }^{1} \mathrm{H}$ and ${ }^{13} \mathrm{C}$ NMR spectra were recorded (as indicated) on either a Bruker 300, 400, or $500 \mathrm{MHz}$ spectrometer and are reported as chemical shifts in ppm relative to TMS $(\mathrm{f1}=0)$. Spin multiplicities are reported as a singlet $(\mathrm{s})$, doublet $(\mathrm{d})$, triplet $(\mathrm{t})$ with coupling constants $(J)$ given in $\mathrm{Hz}$, or multiplet $(\mathrm{m})$. Broad peaks are marked as br. HRESI-MS was performed on QSTAR Pulsar (AB/MDS Sciex) spectrometer and are reported as mass-per-charge ratio $\mathrm{m} / \mathrm{z}$. IR spectra were recorded on a PerkinElmer Spectrum One FT-IR spectrometer (ATR, Golden Gate).

Film Balance Measurements. Pressure-area isotherms were recorded on a homemade computer interfaced Langmuir trough with a surface area of $300 \mathrm{~cm}^{2}$. The Wilhelmy method was used to measure the surface tension with a roughened glass plate (circumference of $36 \mathrm{~mm}$ ) with an accuracy of $\pm 0.1 \mathrm{mN} / \mathrm{m}$. Each measurement was repeated at least three times. The Langmuir trough was filled with ultrapure water (specific resistance of $18.2 \mathrm{M} \Omega \cdot \mathrm{cm}$ ). Stock solutions $(1 \mathrm{mM})$ of the lipids $2,3,5$, and 6 in chloroform and of the lipids 1 and 4 in a 9:1 $\mathrm{CHCl}_{3} / \mathrm{MeOH}$ mixture were used for the spreading process. In order to let the system equilibrate and for complete solvent evaporation, the compression $\left(5 \AA^{2} / \mathrm{molecule} / \mathrm{min}\right)$ was started 5-15 min (depending on the temperature) after spreading the lipid solution.

Grazing Incidence X-ray Diffraction Measurements. Crystalline structures in condensed monolayers at the air/water interface were investigated by grazing incidence X-ray diffraction measurements at the BW1 beamline, HASYLAB (DESY, Hamburg, Germany). ${ }^{17-20}$ At BW1, a monochromatic X-ray beam $(\lambda=1.304 \AA)$ struck the water surface at a grazing incidence angle $\alpha_{\mathrm{i}}=0.85 \alpha_{\mathrm{c}}$ (the critical angle for total external reflection $\alpha_{\mathrm{c}} \sim 0.13^{\circ}$ ) and illuminated roughly $100 \mathrm{~mm}^{2}$ of the monolayer surface. During the course of an experiment, the trough was laterally moved to avoid sample damage by the strong $\mathrm{X}$ ray beam. A linear position-sensitive MYTHEN detector system (PSI, Villigen, Switzerland) measured the diffracted signal and was rotated to scan the in-plane $Q_{x y}$ component values of the scattering vector. A Soller collimator in front of the MYTHEN restricted the in-plane divergence of the diffracted beam to $0.09^{\circ}$. The vertical strips of the MYTHEN measure the out-of-plane $Q_{z}$ component of the scattering vector between 0.0 and $0.75 \AA^{-1}$. The intensities of the scattered radiation were corrected for polarization, footprint area, and powder averaging. Model peaks taken to be Lorentzian in the in-plane direction (Bragg peak) and Gaussian in the out-of-plane direction (Bragg rod) were fitted to the corrected intensities. The in-plane lattice repeat distances $d$ of the ordered structures in the monolayer were calculated from the Bragg peak positions, $d=2 \pi / Q_{x y}$. To access the extent of the crystalline order in the monolayer, the in-plane coherence length, $L_{x y}$, was approximated from the full-width at halfmaximum (fwhm) of the Bragg peaks using $L_{x y} \sim 0.9(2 \pi) / \mathrm{fwhm}\left(Q_{x y}\right)$. The thickness of the monolayer can be estimated from the fwhm of the Bragg rod using $0.9(2 \pi) / \mathrm{fwhm}\left(Q_{z}\right)$.

Computational Methods. The considered cluster models of the layer consisted of dimers, tetramers, and hexamers of either urea or thiourea compounds in the gas phase. Density functional theory method (B3LYP and 6-31G* basis set as implemented in the Turbomole package) was used to determine the most stable conformer of each considered cluster. The smaller models (dimers and tetramers) were used only to verify the adequacy of the procedure applied in the principal study of the hexamers. For either considered hexamers, the search for the most stable conformer was limited to the hydrogenbonded heads. The geometry of all carbons in the aliphatic chains except for the two near the urea or thiourea tail was frozen at the position determined using second order Møller-Plesset calculation. For details see Supporting Information.

\section{RESULTS AND DISCUSSION}

The amide (3), urea $(1,4)$, and thiourea $(2,5,6)$ amphiphiles (Figure 1) were synthesized using new or established protocols

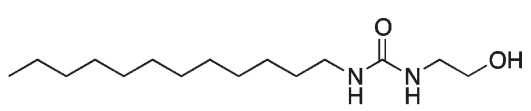

1

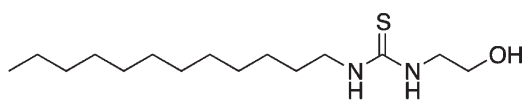

2<smiles>CCCCCCCCCCCCCCCCC(=O)NCCO</smiles><smiles>CCCCCCCCCCCCCCCNC(=O)NCCO</smiles><smiles>CCCCCCCCCCCCCCCNC(=S)NCCO</smiles>

5<smiles>CCCCCCCCCCCCCCCCNC(=S)NCCO</smiles>

Figure 1. Chemical structures of the amide, urea, and thiourea monoalkyl amphiphiles synthesized for this study.

(see Supporting Information). The amide (3) was prepared by coupling ethanolamine to the corresponding long-tailed activated fatty acid. The coupling of ethanolamine to longtailed isocyanates or isothiocyanates yielded the ureas $(1,4)$ or thioureas $(2,5,6)$, respectively (see Supporting Information for details).

The melting points of all compounds are presented in Table 1. Comparing the melting points of compounds with the same

Table 1. Comparison of the Melting Points of the Amide, Urea, and Thiourea Compounds

\begin{tabular}{cccc} 
chain & amide & urea & thiourea \\
$\mathrm{C} 14$ & $90-91{ }^{\circ} \mathrm{C}^{1}$ & $103-105{ }^{\circ} \mathrm{C}$ & $66-68{ }^{\circ} \mathrm{C}$ \\
$\mathrm{C} 17$ & $99-101{ }^{\circ} \mathrm{C}$ & $112-113^{\circ} \mathrm{C}$ & $75-77^{\circ} \mathrm{C}$ \\
$\mathrm{C} 18$ & $106-108{ }^{\circ} \mathrm{C}^{10}$ & & $80-81{ }^{\circ} \mathrm{C}$ \\
\hline
\end{tabular}

hydrophobic chain pattern and therefore the same van der Waals interactions a large influence of the hydrogen-bonding motive can be observed. Clearly, the ureas show the highest melting points. The corresponding amides exhibit $10{ }^{\circ} \mathrm{C}$ lower melting temperatures and the thioureas the lowest ones demonstrating that the essential factor for these differences arises from the chemical headgroup structure. These findings corroborate earlier research on crystal engineering with these moieties. ${ }^{13}$ As observed for many compounds with alkyl chains, the melting points within a homologous series do not show a monotonic behavior with increasing chain length but an alternation with relatively higher melting temperatures for the 

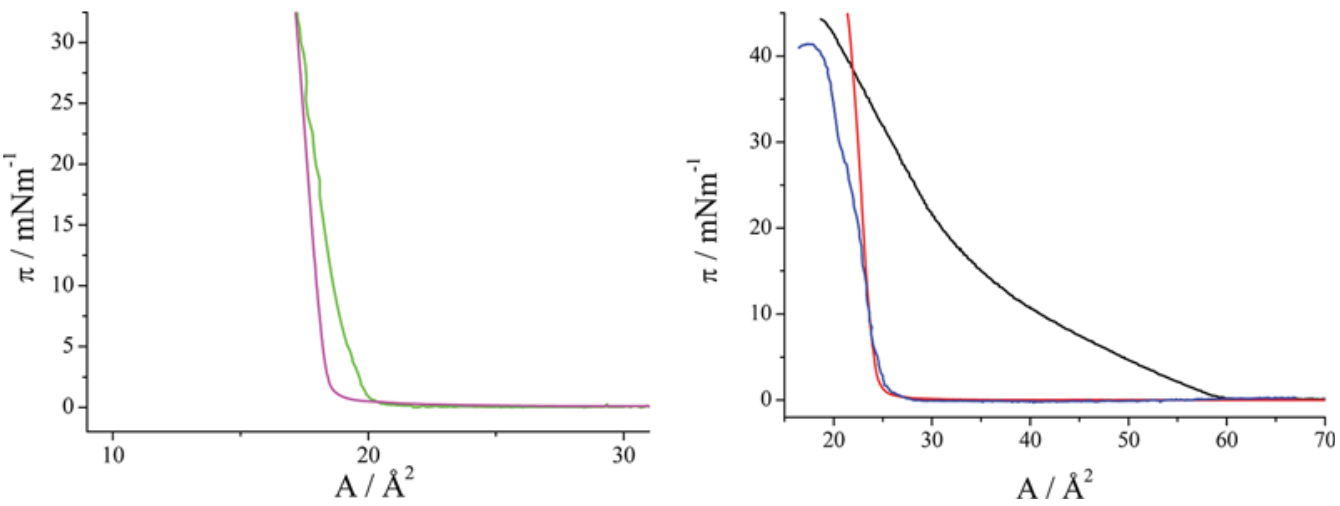

Figure 2. Left: Lateral pressure $\pi$ / molecular area A isotherms of urea (1, green; 4, magenta) and thiourea (2, black; 5, red; 6, blue) amphiphiles at $20{ }^{\circ} \mathrm{C}$ on water. It is worth mentioning that the molecular areas in the condensed state of $\mathbf{1}$ and $\mathbf{4}$ are extremely small most probably due to the formation of small 3D crystallites during spreading.

members with even carbon numbers. ${ }^{21}$ Packing effects are the explanation for such an alternation.

The pressure/area $(\pi / \mathrm{A})$ isotherms are difficult to measure using a Wilhelmy plate as pressure sensor (see Supporting Information for detailed discussion). Therefore, the isotherms are used only for qualitative analysis (Figure 2). As expected from the generic behavior in homologous series of amphiphiles, the influence of increasing chain length is equivalent to decreasing temperature and leads to the disappearance of the LE phase. $^{22,23}$

The amide 3 (Supporting Information Figure S14) does not exhibit a LE phase up to $30{ }^{\circ} \mathrm{C}$. This can be easily understood comparing the results with those of the corresponding shorterchain amide ( $N$-(2-hydroxyethyl) tetradecanamide $)^{1}$ for which the LE phase disappeared below $7^{\circ} \mathrm{C}$. Therefore, the extension of the hydrophobic part by one methylene group corresponds to a shift in temperature of at least $8{ }^{\circ} \mathrm{C}$. The influence of the headgroup structure on the ordering of the amphiphiles 3, 4, and $\mathbf{5}$ with the same alkyl chain length is difficult to predict in a straightforward way. For example, the change from 2hydroxyethyl (compound 3) to methylbutanoate ${ }^{16}$ does not change the phase behavior considerably. The influence of the headgroup on the ordering in monolayers becomes more important if additional strong forces appear. The two urea derivatives 1 and 4 do not show a LE phase and exhibit apparently too small molecular areas $\left(<17 \AA^{2}\right)$ in the condensed state. Excluding such obvious sources of errors as concentration and spread amount, the direct formation of condensed islands could be connected with the formation of small 3D crystallites during the spreading process due to concentration fluctuations and the existence of additional strong attractive interactions. The different behavior of the urea and amide derivatives is most probably caused by the formation of a stronger hydrogen bond network between the urea head groups. Dreger et al. estimated the strength of hydrogen bonds for the amide group. ${ }^{16}$ The strength is approximately the same in both LE and LC phases. The situation is different for the urea derivatives, the energy for the $\mathrm{H}$-bridges is clearly higher in the LC phase compared to the LE phase. ${ }^{16}$ This difference is in line with the experimental observation of the high stability of LC phases compared to LE phases for the corresponding urea derivatives. The comparison of the urea derivative $\mathbf{1}$ with the corresponding more fluid thiourea derivative $\mathbf{2}$ is in line with the idea of weaker hydrogen bonds due to the reduced electronegativity of the sulfur atom. On the basis of only isotherm measurements, one can conclude that the amide and thiourea derivatives $\mathbf{3}$ and $\mathbf{5}$ form hydrogen bond networks weaker than the one of the urea derivative 4 , and the weakest is formed by thiourea.

Grazing incidence X-ray diffraction (GIXD) measurements have been performed in order to identify at an angstrom level the structural parameters of the condensed monolayers of urea 4 and thiourea 5 . The condensed phase structure of amide 3 has not been measured but should be, based on the generic phase behavior concept, ${ }^{18}$ the $\mathrm{L}_{2}{ }^{\prime}$ phase with a cross-sectional area of only $18.8 \AA^{2}$. The $\mathrm{L}_{2}{ }^{\prime}$ phase is characterized by an orthorhombic structure with NNN tilted alkyl chains (herringbone packing mode in the lattice perpendicular to the chain long axes) as determined for the corresponding compound with the shorter chain (C14, N-myristoylethanolamine (tetradecanoic acid-(2-hydroxyethyl)amide)). ${ }^{1}$

Similar results have been found for thiourea 5. Only marginal changes of the well-defined monolayer structure can be observed on compression along the $\pi / \mathrm{A}$-isotherm and at different temperatures of the subphase. In Figure $3 \mathrm{~A}$, the corrected X-ray intensities are presented as a function of the inplane scattering vector component $Q_{x y}$ and the out-of-plane scattering vector component $Q_{z}$. A detailed analysis of the data revealed that the lattice is defined by three main diffraction peaks. Actually, the Bragg peak appearing at higher $Q_{x y}$ and lower $Q_{z}$ values is composed of two strongly overlapping diffraction peaks with the same $Q_{x y}$ but different $Q_{z}$ values. Such scattering intensity distribution is unusual and was not observed before. The analysis of the corresponding Bragg peaks and Bragg rods and the structural parameters are presented in Tables S1 and S2 (Supporting Information). The chains are tilted to the normal by $33^{\circ}$ in the direction of the next-nearest neighbors. The rigidity of the monolayer (strongly tilted Wilhelmy plate on compression if a small paper plate was used) and the fixed structure indicate strong interactions between the head groups due to the formation of a hydrogen bond network.

Moreover, the Bragg rods are characterized by a full-width at half-maximum (fwhm) of $0.28 \AA^{-1}$, which correponds to a scattering length of $20.2 \AA$. This value agrees well with the length of the extended alkyl chain in all-trans conformation. The observation confirms that the interfacial layer is indeed a monolayer at both low and high lateral pressures, because multilayers would generate Bragg rods with significantly smaller fwhm. ${ }^{24,25}$ 

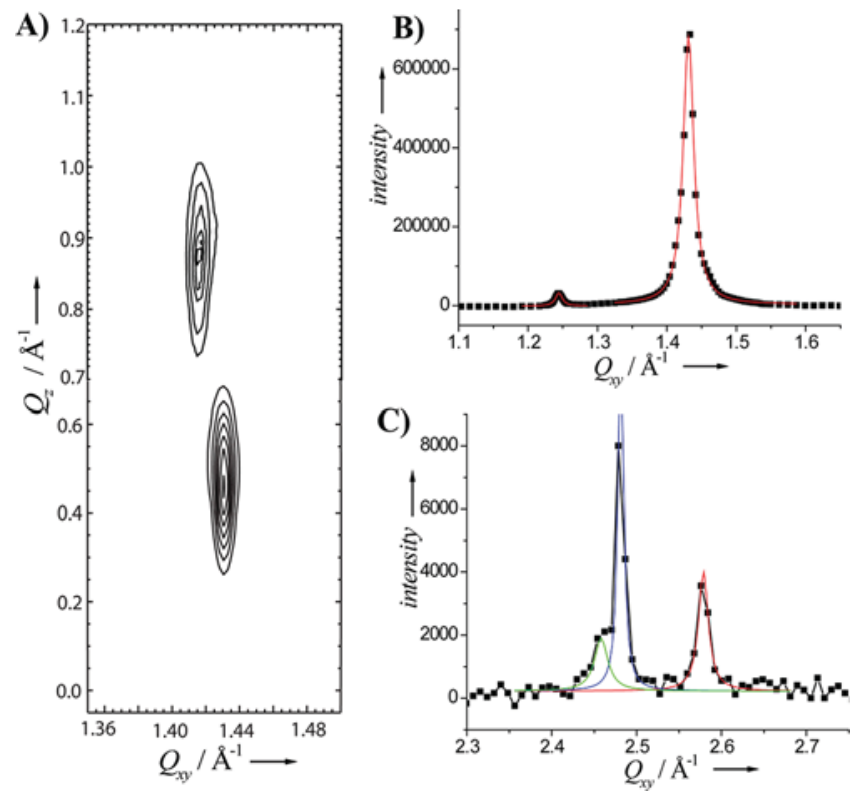

Figure 3. (A) Representative contour plot of corrected X-ray intensities as a function of the in-plane $\left(Q_{x y}\right)$ and out-of-plane $\left(Q_{z}\right)$ scattering vector components of monolayers of thiourea $\mathbf{5}$ on water at $20{ }^{\circ} \mathrm{C}$; $(\mathrm{B}, \mathrm{C})$ Integrated $\mathrm{X}$-ray intensities versus the in-plane scattering vector component $Q_{x y}$ of thiourea 5 monolayers and the corresponding Lorentz fits.

Additionally to the described diffraction pattern, further Bragg peaks have been measured (Figure 3B,C) indicating a highly ordered LC phase. These peaks belong to the same orthorhombic in-plane lattice and are indexed in Supporting Information Table S3. The extremely small cross-sectional area of $18.9 \AA^{2}$ indicates that the molecules are in a "frozen" structure with no possibility for rotation. The dimensions of the lattice perpendicular to the chain long axes are with $a_{\mathrm{r}}=5.057$ $\AA$ and $b_{\mathrm{r}}=7.484 \AA$ typical for a herringbone packing mode. ${ }^{17,26}$ These diffractions peaks indicate the existence of a highly ordered molecular lattice due to a hydrogen bond network as previously reported for a double-chain GPI-fragment. ${ }^{27}$

One additional important experimental observation should be discussed, namely the appearance of a nonequilibrium structure immediately after lateral compression of the thiourea 5 monolayers at high surface pressures $(15,30 \mathrm{mN} / \mathrm{m})$. The contour plot of this temporary structure is represented in
Figure 4A. The new monolayer structure is characterized by a different chain tilt direction (to the nearest neighbors) and a clearly smaller tilt angle of the alkyl chains $\left(22^{\circ}\right.$ compared to $33^{\circ}$ ) (see Supporting Information Tables S4 and S5). The orthorhombic lattice structure leads to a cross-sectional area of $20.0 \AA^{2}$ showing that the molecules, in contrast to the previously described structure, are freely rotating. This behavior can be understood in terms of breaking the headgroup interactions and the formed hydrogen bond network giving rise to the observed molecule rotation. The temporary structure transforms in a self-healing process in a rather short time $(\sim 20$ $\min$ ) into the equilibrium structure presented above (Figure 3 ).

For comparison, GIXD measurements have also been performed with urea 4 monolayers. As in the case of thiourea 5 , the monolayer structure does not respond to changes in the surface pressure or the subphase temperature. A representative contour plot is presented in Figure 5. The monolayers of urea 4 exhibit an orthorhombic structure (Supporting Information Tables $S 6$ and $S 7)$ characterized by two Bragg peaks $\left(Q_{x y}=\right.$ $\left.1.441 \AA^{-1}, Q_{z}=0 \AA^{-1}\right)$ and $\left(Q_{x y}=1.337 \AA^{-1}, Q_{z}=0.958 \AA^{-1}\right)$. The structure is therefore different compared to thiourea 5 . The molecules are now tilted into the direction of the nearest neighbors (NN) and extremely tightly packed with an even smaller cross-sectional area of only $18.5 \AA^{2}$. The dimensions of the lattice perpendicular to the chain long axes are with $a_{\mathrm{r}}=$ $4.267 \AA$ and $b_{\mathrm{r}}=8.72 \AA$ typical for a pseudoherringbone packing mode. The determined values of the fwhm of the Bragg rods $\left(0.27 \AA^{-1}\right)$ are typical for a monolayer, as described above for the thiourea 5 .

In addition, the GIXD data of the urea 4 monolayer reveal scattering intensity distributed on Scherrer rings indicating the formation of some 3D crystallites. The small crystallites are not the result of the compression process but they are already formed during spreading due to density fluctuations. The intensities of the diffraction peaks (2D and 3D phases) increase with increasing lateral pressure. The formation of such crystallites explains the observed too small molecular areas in the compression isotherm. Comparing the in-plane area determined by GIXD and the molecular area from the isotherm shows that $\sim 30 \%$ of molecules form the crystallites. This is in good agreement with a rough estimation comparing the GIXD intensities that gives a value of $23 \%$.

A comparison of the packing properties of thiourea 5 and urea 4 monolayers indicates the existence of a stronger hydrogen bond network in the urea derivative monolayer.
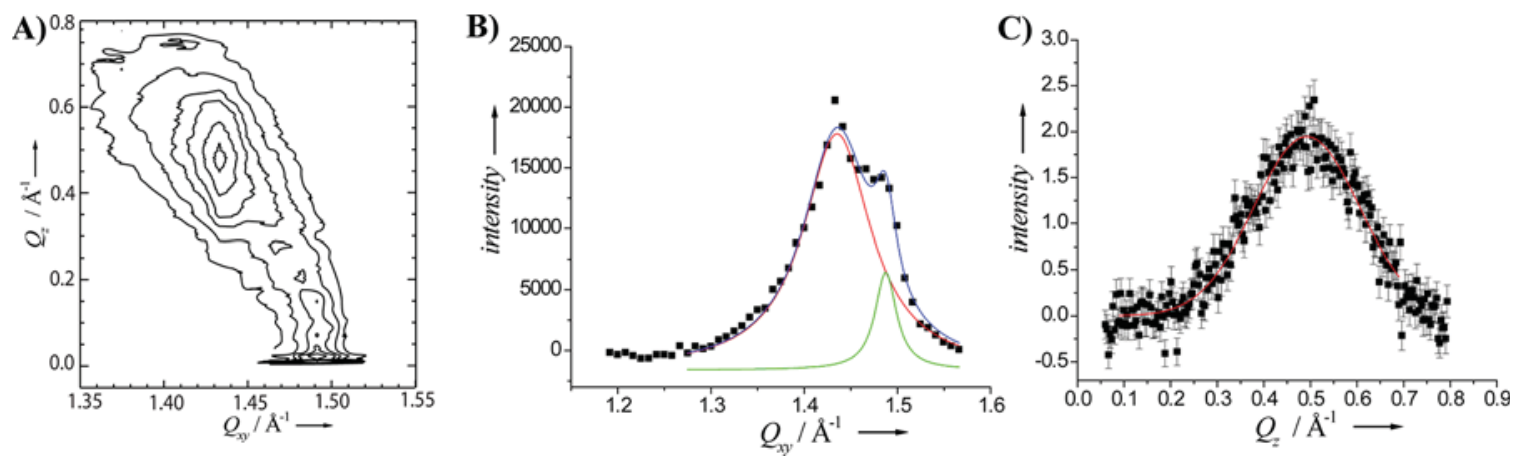

Figure 4. (A) Representative contour plot of corrected $X$-ray intensities as a function of the in-plane $\left(Q_{x y}\right)$ and out-of-plane $\left(Q_{z}\right)$ scattering vector components of the structure of thiourea 5 monolayers on water at $30 \mathrm{mN} / \mathrm{m}$ and $10{ }^{\circ} \mathrm{C}$. (B) Integrated X-ray intensities versus the in-plane scattering vector component $Q_{x y}$ (Bragg peaks) fitted with a superposition of two Lorenz functions and (C) the Bragg rod of the degenerated Bragg peak (fitted with a Gauss function). 


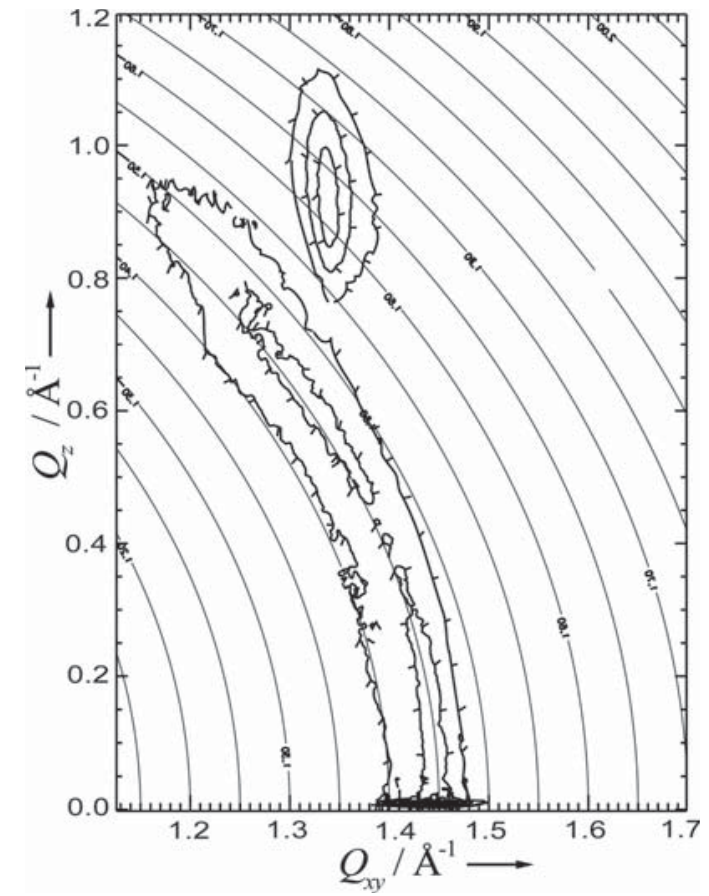

Figure 5. Representative contour plot of corrected X-ray intensities as a function of in-plane $\left(Q_{x y}\right)$ and out-of-plane $\left(Q_{z}\right)$ scattering vector components of the monolayer structure of urea 4 on water at $30 \mathrm{mN} /$ $\mathrm{m}$ and $10{ }^{\circ} \mathrm{C}$. The Scherrer ring indicates the formation of $3 \mathrm{D}$ crystallites giving raise to the too small molecular areas in the isotherms in Figure 2, left.

Thus, due to the rigid interconnections established between the head groups the maximization of the van der Waals interactions leads to an important tilting $\left(40^{\circ}\right)$ of the alkyl chains in respect to the normal to the interface.

Interestingly, the presence of crystallites shows again that the hydrogen bond network in urea $\mathbf{4}$ monolayers is stronger than the one in the thiourea $\mathbf{5}$ monolayers. Additionally, this behavior corroborates beautifully the fact that the hydrogen bonds in the thiourea $\mathbf{5}$ monolayer can be broken upon compression. The nonequilibrium monolayer structure is characterized by the free rotation of molecules. As presented above, the equilibrium monolayer structure (hydrogen bond network between the head groups) is reformed after a short time. Thus, the hydrogen bonds formed in the thiourea 5 monolayers seem to have an "elastic" behavior, being able to be destroyed and reformed, while the ones formed in the urea 4 monolayers are extremely rigid. One might speculate that the amide derivates behave similar to the thiourea derivates but with a much shorter existing time of the intermediate rotator phase.

To gain a better understanding of the headgroup interactions and differences between thiourea and urea moieties, the geometry of a hexamer of the corresponding head groups was optimized using DFT-based calculations. A hexamer is the minimal cluster allowing for all hydrogen bonding possibilities involving $\mathrm{C}=\mathrm{O}$ or $\mathrm{C}=\mathrm{S}$ groups as acceptors (see Supporting Information Figure S15). In principle, several arrangements of $\mathrm{C}=\mathrm{O} \cdots \mathrm{HN}($ or $\mathrm{C}=\cdots)$ motifs are possible leading to many local minima. The lowest energy minimum was found by successive geometry minimizations and eventually replacements of the carbonyl oxygen by sulfur (see Supporting Information). These geometries show a striking difference between the urea and thiourea motifs. In the case of urea, the hydrogen bonds involve a common acceptor $(\mathrm{CO})$ and two donors $(\mathrm{NH})$ of the neighboring headgroup in the same "row". The $180^{\circ}$ arrangement between two urea moieties leads to the formation of distinctive "rows" of molecules. The two $\mathrm{O}-\mathrm{N}$ distances are the same $(2.98 \AA)$ and the two small $\mathrm{O}-\mathrm{H}$ distances $(2.04$ and $2.06 \AA$ A) indicate hydrogen bonding. Additionally, hydrogen bonds can be formed between the OH-groups at the very end of the headgroup. The $\mathrm{O}-\mathrm{N}$ distances to the other two nearest $\mathrm{NH}$ donors are much longer (more than $5 \AA$ ). The hydrogen bonds are symbolically represented by dashed lines connecting head groups of the same "row" (Figure 6A).
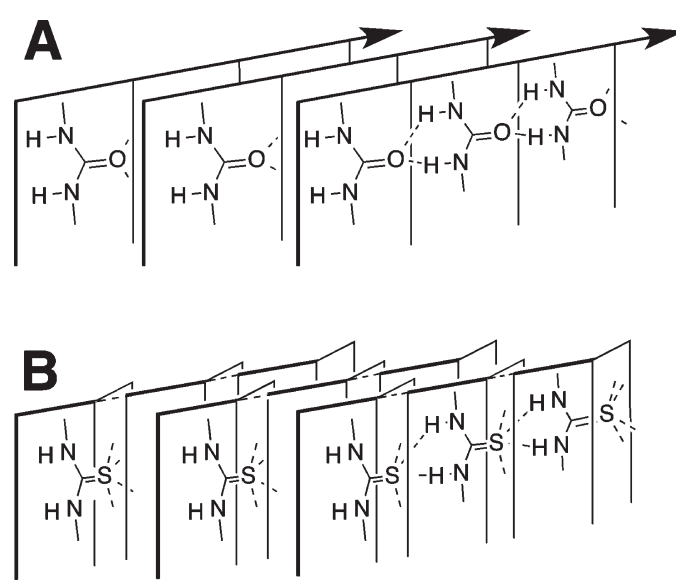

Figure 6. Schematic representation of the calculated results showing lines of hydrogen-bonded urea head groups (A) and interconnected networks of thiourea head groups (B). The thiourea head groups are turned away from the $180^{\circ}$ linear arrangement and can therefore undergo possible hydrogen bonding with more than one direct neighbor.

The situation is qualitatively different in the case of a hexamer consisting of thioureas. Here, the distances between sulfur as hydrogen bond acceptor and nitrogens that are potential hydrogen bonding donors are equal to $3.35,4.24$ (same "row"), 3.82, and $3.56 \AA$ ("neighboring 'row"). Only one hydrogen bond linking the thioureas of the same "row" is energetically favorable. These $\mathrm{H}-\mathrm{S}$ and $\mathrm{N}-\mathrm{S}$ distances are equal to 2.37 and $3.35 \AA$, respectively. The other $\mathrm{N}-\mathrm{H} \cdots \mathrm{S}=\mathrm{C}$ arrangement linking the head groups of the same layer cannot be expected to provide any considerable stabilizing effect because the corresponding $\mathrm{H}-\mathrm{S}$ and $\mathrm{N}-\mathrm{S}$ distances are 3.53 and $4.24 \AA$, respectively. This is the least favorable structural arrangement among the four possible $\mathrm{N}-\mathrm{H} \cdots \mathrm{S}=\mathrm{C}$ motifs. However, the arrangement of the other two $\mathrm{N}-\mathrm{H} \cdots \mathrm{S}=\mathrm{C}$ motifs, connecting the head groups of parallel "rows", can be expected to provide more significant stabilizing effect. One of them could be interpreted as a weak hydrogen bond with the $\mathrm{H}-\mathrm{S}$ and $\mathrm{N}-\mathrm{S}$ distances equal to 3.14 and $3.56 \AA$, respectively, and the other as even a weaker one with $\mathrm{H}-\mathrm{S}$ and $\mathrm{N}-\mathrm{S}$ distances equal to 3.35 and $3.82 \AA$, respectively. These weak specific "out-of-plane" interactions linking the head groups of different "rows" are indicated in Figure 6B.

The most important difference in the conformation of the heads in thiourea and urea clusters is therefore a different arrangement of the hydrogen bonds leading to different inplane lattice structures. In the urea case, the carbonyl groups seem to prefer an aligned (linear network) conformation whereas thiourea forms a true $2 \mathrm{D}$ network (Figure 6). The 
sulfur atom is located between the planes whereas the corresponding oxygen in the urea case lies more in the plane. Separate layers stabilized by hydrogen bonds are distinguishable in the urea case. This observation is in line with the results reported by Custelcean. ${ }^{13}$ Concerning the interaction energy, the urea clusters are energetically more favorable than the thiourea ones $(-67.8 \mathrm{kcal} / \mathrm{mol}$ vs $-34.7 \mathrm{kcal} / \mathrm{mol})$. Compression leads to a temporary rupture of the $2 \mathrm{D}$ hydrogen bond network in the thiourea ethanolamine monolayers, whereas the urea planes can slide during compression keeping the monolayer structure intact.

\section{CONCLUSIONS}

In conclusion, a series of amido-, urea-, and thiourea amphiphiles with varying alkyl chain lengths was synthesized. The surface pressure/molecular area isotherms showed the expected temperature and chain-length dependence as observed for other amphiphiles. A balance between the van der Waals forces and the strength of the hydrogen bonds between the head groups determines the monolayer structure. The simulations using a hexamer cluster model of the head groups indicate a qualitative difference in the $2 \mathrm{D}$ arrangement of the hydrogen bond networks. The hydrogen bonds in the thiourea case form a $2 \mathrm{D}$ network, which ruptures temporarily during compression and is recovered in a self-healing process, while in the urea clusters the hydrogen bonds form a more planar framework with gliding planes keeping the structure intact during compression. Therefore, thiourea-based monolayers are clearly more elastic with a self-healing behavior compared to the more rigid urea-based films.

\section{ASSOCIATED CONTENT}

\section{S Supporting Information}

Synthesis and NMR spectra, pressure/area $(\pi / \mathrm{A})$ isotherms, GIXD data and lattice parameters, computational strategy, and lowest-energy hexamer structures.

\section{AUTHOR INFORMATION}

\section{Corresponding Authors}

*E-mail: tomasz.wesolowski@unige.ch.

*E-mail: brezesinski@mpikg.mpg.de.

*E-mail: andreas.zumbuehl@unifr.ch.

\section{Author Contributions}

The manuscript was written through contributions of all authors.

\section{Notes}

The authors declare no competing financial interest.

\section{ACKNOWLEDGMENTS}

A.Z. thanks the Swiss National Science Foundation (Grant P00P2_138926/1) and the NCCR for Chemical Biology. C.S. thanks the Max Planck society for a fellowship. We thank Irina Berndt for careful isotherm measurements and HASYLAB at DESY, Hamburg (Germany) for beamtime and excellent support.

\section{REFERENCES}

(1) Brezesinski, G.; Dobner, B.; Stefaniu, C.; Vollhardt, D. Monolayer Characteristics of an $\mathrm{N}$-acylated ethanolamine at the air/ water interface. J. Phys. Chem. C 2011, 115, 8206-8213.
(2) Petrosino, S.; Iuvone, T.; Di Marzo, V. N-palmitoyl-ethanolamine: Biochemistry and new therapeutic opportunities. Biochimie 2010, 92, 724-727.

(3) Hansen, H. S.; Moesgaard, B.; Hansen, H. H.; Petersen, G. NAcylethanolamines and precursor phospholipids - relation to cell injury. Chem. Phys. Lipids 2000, 108, 135-150.

(4) Rudert, R.; Wu, D.; Vollhardt, D. The crystal structure of N-(3hydroxypropyl)hexadecanamide. Z. Kristallogr. 1996, 211, 114-116.

(5) Rudert, R.; André, C.; Wagner, R.; Vollhardt, D. A consideration of the hydrogen bonding schemes of the surfactant N-tetradecyl-(2, 4dihydroxy)-butanoic acid amide and some related amphiphilic compounds. Z. Kristallogr. 1997, 212, 752-755.

(6) Ramakrishnan, M.; Swamy, M. J. Molecular packing and intermolecular interactions in $\mathrm{N}$-acylethanolamines: crystal structure of N-myristoylethanolamine. Biochim. Biophys. Acta 1999, 1418, 261267.

(7) Kamlekar, R. K.; Swamy, M. J. Molecular packing and intermolecular interactions in two structural polymorphs of $\mathrm{N}$ palmitoylethanolamine, a type 2 cannabinoid receptor agonist. $J$. Lipid Res. 2006, 47, 1424-1433.

(8) Holme, M. N.; Fedotenko, I. A.; Abegg, D.; Althaus, J.; Babel, L.; Favarger, F.; Reiter, R.; Tanasescu, R.; Zaffalon, P.-L.; Ziegler, A.; Müller, B.; Saxer, T.; Zumbuehl, A. Shear-stress sensitive lenticular vesicles for targeted drug delivery. Nat. Nanotechnol. 2012, 7, 536543.

(9) Coe, S.; Kane, J. J.; Nguyen, T. L.; Toledo, L. M.; Winninger, E.; Fowler, F. W.; Lauher, J. W. Molecular Symmetry and the Design of Molecular Solids: The Oxalamide Functionality as a Persistent Hydrogen Bonding Unit. J. Am. Chem. Soc. 1997, 119, 86-93.

(10) Pal, A.; Dey, J. Water-induced physical gelation of organic solvents by $\mathrm{N}$-(n-alkylcarbamoyl)-L-alanine amphiphiles. Langmuir 2011, 27, 3401-3408.

(11) Bordwell, F. G.; Algrim, D. J.; Harrelson, J. A. The Relative Ease of Removing a Proton, a Hydrogen-Atom, or an Electron from Carboxamides versus Thiocarboxamides. J. Am. Chem. Soc. 1988, 110, 5903-5904.

(12) Gregoret, L. M.; Rader, S. F.; Fletterick, R. F.; Cohen, F. E. Hydrogen bonds involving sulfur atoms in proteins. Proteins 1991, 9 (2), 99-107.

(13) Custelcean, R. Sulfate separation by selective crystallization of a urea-functionalized metal-organic framework. Chem. Commun. 2007, 295-307.

(14) Brezesinski, G.; Möhwald, H. Langmuir monolayers to study interactions at model membrane surfaces. Adv. Colloid Interface Sci. 2003, 100-102, 563-584.

(15) Eeman, M.; Deleu, M. From biological membranes to biomimetic model membranes. Biotechnol. Agron. Soc. Environ. 2010, $14,719-736$

(16) Dreger, K.; Zou, B.; Mu, Z.; Galla, H. J.; Chi, L.; Fuchs, H.; Schäfer, H. J. Synthesis and Surface Properties of New Ureas and Amides at Different Interfaces. Langmuir 2006, 22, 1619-1625.

(17) Kaganer, V. M.; Möhwald, H.; Dutta, P. Structure and phase transitions in Langmuir monolayers. Rev. Mod. Phys. 1999, 71, 779819.

(18) Bringezu, F.; Dobner, B.; Brezesinski, G. Generic phase behavior of branched-chain phospholipid monolayers. Chem.-Eur. J. 2002, 8, 3203-3210.

(19) Kjaer, K. Some simple ideas on X-ray reflection and grazingincidence diffraction from thin surfactant films. Physica B 1994, 198, $100-109$.

(20) Stefaniu, C.; Brezesinski, G. X-ray investigation of monolayers formed at the soft air/water interface. Curr. Opin. Colloid Interface Sci. 2014, 19, 216-227.

(21) Boese, R.; Weiss, H.-C.; Bläser, D. The melting point alternation in the short-chain n-alkanes: Single-crystal X-ray analyses of propane at $30 \mathrm{~K}$ and of n-butane to n-nonane at $90 \mathrm{~K}$. Angew. Chem., Int. Ed. 1999, 38, 988-992.

(22) Albrecht, O.; Gruler, H.; Sackmann, E. Polymorphism of phospholipid monolayers. J. Phys. Paris 1978, 39, 301-313. 
(23) Glazer, J.; Alexander, A. E. The monolayer polymorphism of hexadecyl and octadecyl urea. Trans. Faraday Soc. 1951, 47, 401-409. (24) Kuzmenko, I.; Kindermann, M.; Kjaer, K.; Howes, P. B.; AlsNielsen, J.; Granek, R.; von Kiedrowski, G.; Leiserowitz, L.; Lahav, M. Crystalline Films of Interdigitated Structures Formed via AmidiniumCarboxylate Interactions at the Air-Water Interface. J. Am. Chem. Soc. 2001, 123, 3771-3783.

(25) Rapaport, H.; Kuzmenko, I.; Lafont, S.; Kjaer, K. Cholesterol monohydrate nucleation in ultrathin films on water. Biophys. J. 2001, 81, 2729-2736.

(26) Kuzmenko, I.; Kaganer, V. M.; Leiserowitz, L. Packing of Hydrocarbon Chains and Symmetry of Condensed Phases in Langmuir Monolayers. Langmuir 1998, 14, 3882-3888.

(27) Stefaniu, C.; Vilotijevic, I.; Santer, M.; Varon Silva, D.; Brezesinski, G.; Seeberger, P. H. Subgel phase structure in monolayers of glycosylphosphatidylinositol glycolipids. Angew. Chem., Int. Ed. 2012, 51, 12874-12878. 